\title{
Internet-Einsteigerkurse bei Health-Info-Net
}

\author{
G. von Below
}

Das erste Halbjahr 2001 hat gezeigt, dass Internetkurse weiterhin einem Bedürfnis entsprechen und auf anhaltendes Interesse stossen. Die Kurse der HealthInfo-Net AG (HIN) erfreuen sich grosser Beliebtheit, waren doch 90\% der angebotenen Kurse fast vollständig ausgebucht.

HIN weist bereits mehrjährige Erfahrung als Kursanbieter auf und kann auf die Zufriedenheit von über 1000 Teilnehmerinnen und Teilnehmern zurückblicken.

Die HIN-Internet-Einsteigerkurse sind speziell auf die Bedürfnisse von HIN-Kundinnen/-Kunden und Interessentinnen und Interessenten zugeschnitten. Sie eignen sich sowohl für Neueinsteiger als auch für bisherige Gelegenheitsanwender, die mehr über Internet und Anwendungsmöglichkeiten wissen möchten. Der Einstieg in das Medium Internet sowie auch in die Sicherheitsproblematik wird dadurch wesentlich erleichtert.

Erfahrene Kursleiterinnen und -leiter führen Sie während 4,5 Stunden kompetent und in konzentrierter Form in die Anwendungen der marktführenden Internettools ein und versorgen Sie mit vielen praktischen Tips. Ebenso erhalten Sie wertvolle Informationen zum Internet, zu HIN und der HIN-eigenen Sicherheitssoftware ASAS.

\section{Kursinhalt}

- Allgemeine Informationen zum Internet, zur notwendigen Infrastruktur zu HIN sowie zur Sicherheitssoftware ASAS;

- Chancen und Gefahren im Internet;

- Navigieren im Internet;

- Surfen, suchen, anschauen und downloaden mit dem Browser Internet Explorer;

- Kennenlernen einer Auswahl der nützlichsten Suchmaschinen; für effizientes Suchen und Finden im Internet (allgemeine Suchmaschine, keine speziellen medizinischen Datenbanken);

- E-mail-Einführung unter Verwendung des Mailprogramms Outlook Express;

- Praktische Übungen.
Von den Teilnehmerinnen und Teilnehmern werden PC-Grundkenntnisse (Windows und MS-Office; z.B. Word/Excel) erwartet.

Die Maximalteilnehmerzahl von 10 Personen pro Kurs garantieren eine gute Betreuung durch die/den Kursleiterin/-leiter, wobei auch auf individuelle Fragen eingegangen werden kann. Jedem Teilnehmer steht ein persönlicher Arbeitsplatz mit Laptop zur Verfügung.

Die Kurse werden jeweils an einem Nachmittag oder Samstagmorgen durchgeführt. In der Teilnahmegebühr von Fr. 345.- pro Teilnehmer sind Pausenverpflegung, Kursunterlagen und Mehrwertsteuer bereits inbegriffen.

Für das 4. Quartal 2001 hat HIN noch folgende Kursdaten ausgeschrieben:

\begin{tabular}{|c|c|c|c|c|}
\hline Datum & Ort & Wochentag & Dauer & Kurs-Nr. \\
\hline & & & & \\
\hline 22 & Olt & & 13 & 01-124 \\
\hline 4. Nov. 2001 & Luzern & Samstag & 09.00-14.00 Uhr & $01-125$ \\
\hline
\end{tabular}

Die Seminarräume befinden sich jeweils in nächster Umgebung des örtlichen Bahnhofes.

Für das kommende Jahr plant HIN, das Angebot mit einer Partnerfirma auf spezifische Kurse im Bereich medizinisch-wissenschaftlicher Datenbanken zu erweitern.

Weitere Informationen und Anmeldung zu den ausgeschriebenen Kursdaten online unter http://www.hin.ch oder persönlich über HIN Call Desk 0848830740.

Korrespondenz: Judith Weber Health-Info-Net AG Pflanzschulstrasse 3 CH-8400 Winterthur 